\title{
Journal of Pharmaceutical Sciences
}

\section{Research Article}

Quaternary complexes modified from pDNA and poly-L-lysine complexes to enhance pH-buffering effect and suppress cytotoxicity

Yukinobu Kodama, ${ }^{1}$ Yuiko Yatsugi, ${ }^{1}$ Takashi Kitahara, ${ }^{1}$ Tomoaki Kurosaki, ${ }^{1}$ Kanoko Egashira, ${ }^{2}$ Mikiro Nakashima, ${ }^{2}$ Takahiro Muro, ${ }^{1}$ Hiroo Nakagawa, ${ }^{1}$ Norihide Higuchi, ${ }^{1}$ Tadahiro Nakamura, ${ }^{1}$ Hitoshi Sasaki ${ }^{1}$

${ }^{1}$ Department of Hospital Pharmacy, Nagasaki University Hospital, 1-7-1 Sakamoto, Nagasaki 852-8501, Japan

${ }^{2}$ Department of Pharmacy Practice, Graduate School of Biomedical Sciences, Nagasaki University, Nagasaki, 1-14 Bunkyo-machi, Nagasaki 852-8521, Japan.

Correspondence to: Hitoshi Sasaki

Department of Hospital Pharmacy, Nagasaki University Hospital, 1-7-1 Sakamoto, Nagasaki 852-8501, Japan

Tel.: +81-95-819-7245

Fax: +81-95-819-7251

E-mail: sasaki@nagasaki-u.ac.jp 


\section{ABSTRUCT}

We developed a modified complex of pDNA and poly-L-lysine (PLL) by the addition of poly-L-histidine (PLH) and $\gamma$-polyglutamic acid ( $\gamma$-PGA) to enhance its pH-buffering effect and suppress cytotoxicity. The binary and ternary complexes of pDNA with PLL or/and PLH showed particle sizes of approximately 52-76 nm with cationic surface charge. The ternary complexes showed much higher gene expression than the binary complexes with PLL. The mixed solution of PLL and PLH showed higher buffering capacity than PLL solution. The high gene expression of ternary complexes was reduced by bafilomycin $A_{1}$. These results indicated the addition of PLH to PLL complexes promoted endosomal escape by enhancing the pH-buffering effect. The binary and ternary complexes showed cytotoxicity and blood agglutination because of their cationic surface charge. We therefore developed quaternary complexes by the addition of anionic $\gamma$-PGA, which was reported to decrease the toxicity of cationic complexes. In fact, quaternary complexes showed no cytotoxicity and blood agglutination. Also, quaternary complexes showed higher gene expression than ternary complexes regardless of their anionic surface charge. Quaternary complexes showed selectively high gene expression in the spleen after their intravenous administration. 
Thus, we successfully developed the quaternary complexes with high gene expression and no toxicity.

Keywords: Non-viral gene delivery; Plasmid DNA; Biocompatibility; Biodegradable polymers; Nanoparticles;

Abbreviations: PLL, poly-L-lysine; PLH, poly-L-histidine; $\gamma$-PGA, $\gamma$-polyglutamic acid; BSA, bovine serum albumin; FBS, fetal bovine serum; WST-1, 2-(4-iodophenyl)-3-(4-nitrophenyl)-5-(2,4-disulfophenyl)-2H-tetrazolium, monosodium salt; 1-methoxy PMS, 1-methoxy-5-methylphenazinium methyl sulfate; EDTA, ethylenediaminetetraacetic acid; RLU, relative light units; CPZ, chlorpromazine 


\section{INTRODUCTION}

A wide range of non-viral gene vectors has been developed, such as cationic polymers and cationic lipids, for successful gene therapy. ${ }^{1,2}$ Several advantages of cationic polymers in the process of gene transfection have been reported: condensing pDNA by electrostatic interaction, binding to the cell surface, take up by the endocytotic pathway, and release of pDNA into the cytoplasm. ${ }^{3,4}$ Among cationic polymers, poly-L-lysine (PLL) was one of the first polymers employed for gene transfer, which is considered to have an advantage for clinical use because of its biodegradable peptide structure. At physiological $\mathrm{pH}$, the amino groups of PLL were positively charged and interact electrostatically with negatively charged DNA, which condensed the DNA in the complexes and protected it. ${ }^{5}$ PLL, however, had a couple of disadvantages for clinical use, such as low gene expression and agglutination of blood. ${ }^{6}$

The low gene transfection efficiency of PLL was reported to be due to its lack of an efficient mechanism for endosomal escape. ${ }^{7}$ Generally, non-viral gene vectors that can escape from endosomes express high gene transfection efficiency. According to the results provided by several groups, using a polymer with high buffering capacity, the efficiency of endosomal escape could be increased by increasing the buffering capacity. ${ }^{8,9}$ Gene vectors with buffering capacity lead to osmotic swelling and rupture 
of endosomes, resulting in the release of vectors into the cytoplasm, a phenomenon called the "pH-buffering effect".

Histidine has potential as a modification group for gene vectors because of its biocompatibility and buffering capacity in the $\mathrm{pH}$ range of endosomes. ${ }^{10-13}$ The accumulation of histidine residues inside acidic vesicles can induce the $\mathrm{pH}$-buffering effect, which could enhance the endosomal escape rate of the gene vector. In previous studies, there are several reports of histidine vectors such as histidylated polylysine, ${ }^{14}$ histidine-rich polymers, ${ }^{15}$ and histidine-rich peptides. ${ }^{16}$ Then, we constructed ternary complexes which incorporated poly-L-histidine (PLH) into PLL-based gene vectors (binary complexes) to achieve more efficient gene transfection.

On the other hand, the cationic surface of pDNA complexes with cationic polymers induced cytotoxicity and blood agglutination caused by their strong interaction with the anionic surface of cells and erythrocytes. ${ }^{17}$ Agglutination of the complexes often led to their rapid elimination and adverse effects, such as embolism and inflammatory reactions. ${ }^{18-20}$ Recharging cationic complexes with an anionic compound was reported to be a promising method for overcoming these toxicities. ${ }^{21,22}$ In our previous study, we discovered anionic polymers such as $\gamma$-polyglutamic acid $\left(\gamma\right.$-PGA), ${ }^{23}$ chondroitin sulfate, ${ }^{24}$ and hyaluronic acid, ${ }^{24}$ which decreased the toxicity of cationic complexes 
while maintaining high transgene efficiency. Among them, the addition of $\gamma$-PGA showed the highest gene expression.

In the present study, we developed modified complexes of pDNA and PLL by the addition of PLH and $\gamma$-PGA to enhance their pH-buffering effect and suppress cytotoxicity. We investigated the efficacy and safety of these quaternary complexes as gene vectors in vitro and in vivo. 


\section{MATERIALS AND METHODS}

\section{Chemicals}

Bovine serum albumin (BSA), PLL (mean molecular weight: 22,500), and PLH (molecular weight: $\geq 5,000$ ) were obtained from Sigma-Aldrich Co. LLC. (St. Louis, MO, USA). The $\gamma$-PGA (mean molecular weight: 55,000) was provided by Yakult Pharmaceutical Industry Co., Ltd. (Tokyo, Japan). Fetal bovine serum (FBS) was purchased from Biological Industries Ltd. (Kibbutz Beit Haemek, Israel). RPMI 1640, Opti-MEM I, antibiotics (penicillin $100 \mathrm{U} / \mathrm{ml}$ and streptomycin $100 \mu \mathrm{g} / \mathrm{ml}$ ), and other culture reagents were obtained from GIBCO BRL (Grand Island, NY, USA).

2-(4-Iodophenyl)-3-(4-nitrophenyl)-5-(2,4-disulfophenyl)-2H-tetrazolium, monosodium salt (WST-1) and 1-methoxy-5-methylphenazinium methylsulfate (1-methoxy PMS) were purchased from Dojindo Laboratories (Kumamoto, Japan). YOYO-1 was purchased from Molecular Probes (Leiden, The Netherlands). All other chemicals were of reagent grade.

\section{Construction of pDNA}

pCMV-Luc was constructed by subcloning the Hind III/Xba I firefly luciferase cDNA fragment from the pGL3-control vector (Promega, Madison, WI, USA) into the 
polylinker of the pcDNA3 vector (Invitrogen, Carlsbad, CA, USA). The pDNA was amplified using an EndoFree Plasmid Giga Kit (QIAGEN GmbH, Hilden, Germany). The pDNA was dissolved in $5 \%$ dextrose solution and stored at $-80{ }^{\circ} \mathrm{C}$ until analysis. The pDNA concentration was measured at $260 \mathrm{~nm}$ absorbance and adjusted to $1 \mathrm{mg} / \mathrm{ml}$.

\section{Preparation of binary, ternary, and quaternary complexes}

PLL-PLH mixture was prepared by mixing PLL solution and PLH solution. To prepare binary and ternary complexes, pDNA solution and PLL-PLH mixture were mixed by pipetting thoroughly. The charge ratios of pDNA, PLL, and PLH were 1:8:0 (PLL8-PLH0 complexes), 1:6:2 (PLL6-PLH2 complexes), 1:4:4 (PLL4-PLH4 complexes), 1:2:6 (PLL2-PLH6 complexes), or 1:0:8 (PLL0-PLH8 complexes). To prepare quaternary complexes, $\gamma$-PGA solution was added to PLL6-PLH2 complexes at various charge ratios of 1:6:2:4 (PLL6-PLH2-PGA4 complexes), 1:6:2:6

(PLL6-PLH2-PGA6 complexes), 1:6:2:8 (PLL6-PLH2-PGA8 complexes), and 1:6:2:10 (PLL6-PLH2-PGA10 complexes).

\section{Physicochemical properties and gel retardation of complexes}

The particle sizes and $\zeta$-potentials of several complexes were measured using a 
Zetasizer Nano ZS (Malvern Instruments, Ltd., Malvern, United Kingdom). The number-fractioned mean diameter is shown.

To determine complex formations, $20 \mu \mathrm{L}$ aliquots of complex solutions containing 1 $\mu$ g pNA were mixed with $4 \mu$ l loading buffer (30\% glycerol and $0.2 \%$ bromophenol blue) and loaded onto a 0.8\% agarose gel. Electrophoresis (i-Mupid J; Cosmo Bio, Tokyo, Japan) was carried out at $50 \mathrm{~V}$ in running buffer solution (40 mM Tris/HCl, 40 $\mathrm{mM}$ acetic acid, and $1 \mathrm{mM}$ ethylenediaminetetraacetic acid (EDTA)) for $60 \mathrm{~min}$. The retardation of pDNA was visualized with ethidium bromide staining.

\section{Transfection and cellular uptake experiments}

The mouse melanoma cell line, B16-F10 cells, was obtained from the Cell Resource

Center for Biomedical Research (Tohoku University, Japan). B16-F10 cells were maintained in RPMI 1640 supplemented with 10\% FBS and antibiotics (culture medium) under a humidified atmosphere of $5 \% \mathrm{CO}_{2}$ in air at $37{ }^{\circ} \mathrm{C}$. B16-F10 cells were plated on 24-well plates (Becton-Dickinson and Company, Franklin Lakes, NJ, USA) at a density of $1.0 \times 10^{4}$ cells/well and cultivated in $500 \mu$ l culture medium. In the transfection experiment, the medium was replaced with $500 \mu \mathrm{l}$ Opti-MEM I medium after $24 \mathrm{~h}$ pre-incubation and each complex containing $1 \mu \mathrm{g}$ pCMV-Luc was added to 
the cells and incubated for $2 \mathrm{~h}$. After transfection, the medium was replaced with culture medium and cells were cultured for a further $22 \mathrm{~h}$ under a humidified atmosphere of $5 \% \mathrm{CO}_{2}$ in air at $37^{\circ} \mathrm{C}$. After $22 \mathrm{~h}$ incubation, the cells were washed with PBS and then lysed in $100 \mu \mathrm{l}$ lysis buffer (pH 7.8 and $0.1 \mathrm{M}$ Tris/HCl buffer containing $0.05 \%$ Triton X-100 and 2 mM EDTA). Ten microliters of lysate samples were mixed with $50 \mu \mathrm{L}$ luciferase assay buffer (PicaGene; Toyo Ink Co., Ltd., Tokyo, Japan) and the light produced was immediately measured using a luminometer (Lumat LB 9507; EG \& G Berthold, Bad Wildbad, German). The protein content of the lysate was determined by the Bradford assay (Bio-Rad Laboratories, Inc., Hercules, CA, USA) using BSA as a standard. Absorbance was measured using a microplate reader (Sunrise RC-R; Tecan Japan Co., Ltd., Kanagawa, Japan) at 595 nm. Luciferase activity was indicated as relative light units (RLU) per mg protein.

To visualize the uptake of the complexes, B16-F10 cells were transfected with complexes containing of YOYO-1-labeled pCMV-Luc. After $22 \mathrm{~h}$ incubation, the relative levels of YOYO-1 in the cells were characterized using fluorescence microscopy (200 × magnification).

\section{pH titration}


The pHs of PLL solution (3.9 $\mu \mathrm{M})$ and mixed solution of PLL and PLH were adjusted to 3.0 with $2 \mathrm{M}$ HCL, and then the solutions were titrated with $0.01 \mathrm{M} \mathrm{NaOH}$. The change of $\mathrm{pH}$ was recorded using a pH meter (LAQUA F-72; Horiba Ltd., Kyoto, Japan) and plotted for comparison of the buffering capacity between groups. The molar ratio of PLL to PLH in mixed solution was the same as that of PLL6-PLH2 complexes.

\section{WST-1 assay}

Cytotoxicity tests of various complexes on B16-F10 cells were carried out using a WST-1 commercially available cell proliferation reagent. The WST-1 reagent was prepared (5 mM WST-1 and 0.2 mM 1-methoxy PMS in PBS) and filtered through a $0.22 \mu \mathrm{m}$ filter (Millex-GP; Millipore Co., Bedford, MA, USA) just before the experiments. B16-F10 cells were plated on 96-well plates (Becton-Dickinson and Company) at a density of $3.0 \times 10^{3}$ cells/well in the culture medium. Complexes containing $1 \mu \mathrm{g}$ pDNA in $100 \mu \mathrm{l}$ Opti-MEM I medium were added to each well and incubated for $2 \mathrm{~h}$. After incubation, the medium was replaced with $100 \mu \mathrm{l}$ culture medium and incubated for another $22 \mathrm{~h}$ at $37^{\circ} \mathrm{C}$. The medium was replaced with 100 $\mu \mathrm{l}$ culture medium and $10 \mu \mathrm{l}$ WST-1 reagent was added to each well. The cells were 
incubated for an additional $2 \mathrm{~h}$ at $37^{\circ} \mathrm{C}$, and absorbance was measured at a wavelength of $450 \mathrm{~nm}$ with a reference wavelength of $630 \mathrm{~nm}$, using a microplate reader. The results are shown as the percentage to untreated cells.

\section{Inhibition study}

For determination of the endocytotic pathway, after 23 h pre-incubation, B16-F10 cells were treated with $0.014 \mathrm{mM}$ chlorpromazine (CPZ) as an inhibitor of clathrin-mediated endocytosis, $0.2 \mathrm{mM}$ genistein as an inhibitor of caveolae-mediated endocytosis, or 1 $\mathrm{mM}$ amiloride as an inhibitor of macropinocytosis for $1 \mathrm{~h}$.

On the other hand, to research their release from endosomes, the cells were treated with $100 \mathrm{nM}$ bafiromycin $\mathrm{A}_{1}$ as an ATPase proton pump inhibitor for $1 \mathrm{~h}$.

After treatment, PLL6-PLH2 complexes or PLL6-PLH2-PGA8 complexes were added to the medium containing each inhibitor and incubated for $2 \mathrm{~h}$. After $2 \mathrm{~h}$ transfection, the medium was replaced with culture medium, cells were cultured for a further $22 \mathrm{~h}$ at $37^{\circ} \mathrm{C}$, and then luciferase activities were determined.

\section{Agglutination study}

Erythrocytes from mice were washed three times at $4{ }^{\circ} \mathrm{C}$ by centrifugation at 5,000 
rpm (Kubota 3500; Kubota, Tokyo, Japan) for 5 min and resuspended in PBS. A 2\% (v/v) stock suspension was prepared. Various complexes were added to the erythrocytes (complexes: stock suspension $=1: 1$ ). The suspensions were incubated for 15 min at room temperature. Ten microliters of these suspensions were placed on a glass plate and agglutination was observed by microscopy (400 × magnification).

\section{Animals}

Animal care and experimental procedures were performed in accordance with the Guidelines for Animal Experimentation of Nagasaki University with approval from the Institutional Animal Care and Use Committee. Male ddY mice (5 weeks old) were purchased from Japan SLC (Shizuoka, Japan). After shipping, mice were acclimatized to the environment for at least one day before the experiments.

\section{In vivo study}

Each complex containing $40 \mu \mathrm{g}$ pCMV-Luc at a volume of $250 \mu \mathrm{l}$ per mouse was injected intravenously into mice to examine the transgene efficacy of PLL-PLH complexes and PLL-PLH-PGA complexes. Six hours after the injection, the mice were sacrificed, and the liver, kidney, spleen, heart, and lung were dissected. The 
tissues were washed twice with cold saline and homogenized with lysis buffer. The homogenates were centrifuged at 15,000 rpm (Kubota 3500) for 5 min and the supernatants were used for luciferase assays. Luciferase activity is indicated as RLU per gram of tissue.

\section{Statistical analysis}

Statistical significance between two groups was identified by Student’s t-test.

Multiple comparisons among groups were made by Dunnett’s pairwise multiple comparison t-test. 


\section{RESULTS}

\section{Physicochemical properties and electrophoresis assay of binary and ternary}

\section{complexes}

The particle sizes and $\zeta$-potentials of the binary and ternary complexes are

summarized in Table 1. The complexes showed a particle size of approximately 52-76 $\mathrm{nm}$ and a $\zeta$-potential of approximately $40-48 \mathrm{mV}$.

Complex formations were examined using a gel retardation assay (Fig. 1). Naked pDNA was detected as bands on an agarose gel. No band was detected in the lanes of binary and ternary complexes.

\section{In vitro transfection efficiency and cellular uptake of binary and ternary}

\section{complexes}

B16-F10 cells were transfected with the binary and ternary complexes and luciferase activity was evaluated in the cells. The in vitro transgene efficiencies of the complexes are shown in Fig. 2A. The complexes of pDNA and PLL (PLL8-PLH0 complexes) showed $1.34 \times 10^{6} \mathrm{RLU} / \mathrm{mg}$ protein in transgene efficiency. On the other hand, the gene expressions of ternary complexes were 42.0-fold (PLL6-PLH2 complexes) and 35.7-fold (PLL4-PLH4 complexes) higher than that of binary complexes (PLL8-PLH0 
complexes). PLL2-PLH6 and PLL0-PLH8 complexes showed lower luciferase activity than PLL8-PLH0 complexes.

B16-F10 cells were transfected with PLL8-PLH0, PLL6-PLH2, or PLL0-PLH8 complexes containing YOYO-1-labeled pCMV-Luc to allow the uptake of the complexes to be visualized (Fig. 2B). As a result, many green dots representing YOYO-1 were observed in most of the transfected cells for PLL8-PLH0 and PLL6-PLH2 complexes. On the other hand, PLL0-PLH8 showed low green dots.

\section{pH titration}

With the addition of $0.01 \mathrm{M} \mathrm{NaOH}$, the pHs of PLL solution and mixed solution of PLL and PLH gradually increased. The mixed solution of PLL and PLH had higher buffering capacity between pH 5 and 7 than PLL solution (Fig. 2C).

\section{Physicochemical properties and electrophoresis assay of quaternary complexes}

In the preliminary experiment, the ternary complexes of pDNA (PLL-PLH complexes) showed cytotoxicity and agglutination. Therefore, we prepared quaternary complexes of pDNA (PLL-PLH-PGA complexes) by the addition of $\gamma$-PGA to PLL6-PLH2 complexes. The particle size and $\zeta$-potential of these PLL-PLH-PGA 
complexes are shown in Table 2. While PLL6-PLH2-PGA4 complexes showed agglutination, the complexes with charge ratios of 8 or 10 for $\gamma$-PGA to pDNA showed a particle size of around $100 \mathrm{~nm}$. The addition of $\gamma$-PGA decreased the $\zeta$-potential of complexes in a concentration-dependent manner. PLL-PLH-PGA complexes with charge ratios of 8 or 10 for $\gamma$-PGA to pDNA had an anionic surface charge. Gel retardation assays were employed to examine the formation of the complexes (Fig. 3). Naked pDNA was detected as bands on an agarose gel. On the other hand, PLL-PLH-PGA complexes showed no band of pDNA that was released.

\section{In vitro transfection efficiency of quaternary complexes}

The quaternary complexes containing pCMV-Luc were incubated with B16-F10 cells and the luciferase activity in the cells was determined. The in vitro transgene efficiencies of the complexes at various charge ratios are shown in Fig. 4A. The transgene efficiencies of PLL6-PLH2 complexes showed $5.5 \times 10^{7}$ RLU/mg protein. The luciferase activity of PLL-PLH-PGA complexes was higher than that of PLL6-PLH2 complexes. 


\section{complexes}

For assessment of the endocytotic pathway, we examined the effects of endocytotic inhibitors on the transgene efficiencies of PLL6-PLH2-PGA8 complexes (Fig. 4B). The transfection efficiency of PLL6-PLH2-PGA8 complexes in the presence of CPZ, genistein, and amilirode decreased to $79.7 \%, 56.0 \%$, and $40.6 \%$ of the original efficiency, respectively.

The influence of $100 \mathrm{nM}$ bafiromycin $\mathrm{A}_{1}$ on transfection efficiency of PLL6-PLH2 complexes (Fig. 2D) or PLL6-PLH2-PGA8 complexes (Fig. 4C) was determined to examine endosomal escape. Transgene efficiencies were significantly suppressed by bafiromycin $\mathrm{A}_{1}(\mathrm{P}<0.05)$.

\section{Cytotoxicity and agglutination study of the quaternary complexes}

Each complex was added to B16-F10 cells and cell viability was determined by the WST-1 assay to evaluate cytotoxicity (Fig. 5A). Both PLL8-PLH0 complexes and PLL6-PLH2 complexes showed significantly higher cytotoxicity than the control (P $<0.05$ ). On the other hand, PLL6-PLH2-PGA8 complexes had no cytotoxicity under the same condition.

Also, each complex was incubated with erythrocytes to evaluate agglutination (Fig. 
5B). Severe agglutination was observed in both PLL8-PLH0 complexes and

PLL6-PLH2 complexes. However, no agglutination was observed in

PLL6-PLH2-PGA8 complexes.

\section{In vivo study}

The in vivo transgene efficiencies of PLL8-PLL0 complexes, PLL6-PLH2 complexes, and PLL6-PLH2-PGA8 complexes containing pCMV-Luc were examined in ddY male mice (Fig. 6). Luciferase activities in several tissues were determined $6 \mathrm{~h}$ after intravenous administration of the complexes. The transgene efficiencies of PLL8-PLH0 complexes showed $1.1 \times 10^{5} \mathrm{RLU} / \mathrm{mg}$ protein in the lung. On the other hand, the gene expressions of PLL6-PLH2-PGA8 complexes were lower in the lung (4.7 $\times 10^{4} \mathrm{RLU} / \mathrm{mg}$ protein), and significantly higher in the spleen $\left(3.4 \times 10^{5} \mathrm{RLU} / \mathrm{mg}\right.$ protein) than those of PLL8-PLH0 complexes and PLL6-PLH2 complexes. 


\section{DISCUSSION}

Cationic polymers are one of the most popular non-viral gene vectors due to their excellent ability to compact anionic genes, synthetic controllability, better flexibility achieved simply by varying the chemical composition, molecular weight, and architecture (linear, randomly branched, dendrimer, block, and graft copolymer). ${ }^{25}$ Espcially, polyethylenimine (PEI) and polyamidoamine (PAMAM) dendrimers show high gene expression in vitro and in vivo. ${ }^{26,27}$ These vectors strongly interact with the cell surface, and are well taken up by cells because of their strong cationic surface.

Moreover, these vectors achieve efficient escape from endosomes to cytoplasm via the $\mathrm{pH}$-buffering effect and the gene released from the vector in the cytoplasm expresses protein in the nucleus.

The cationic polymer PLL was reported to show low gene expression because of its lack of pH-buffering effect, regardless its high uptake into the cells. ${ }^{6,7,28}$ Therefore, we tried to develop ternary complexes of pDNA with PLL and PLH, which are polymers with pH-buffering capacity. Histidine of PLH, a non-essential amino acid, contains an imidazole ring which increases the buffering capacity in endosomes and lysosomes. Histidine-containing dipeptides, such as carosine, anserine, and balenine, are also known to act as endogenous buffers in skeletal muscle. ${ }^{11,29}$ 
The binary complexes of pDNA with PLL or PLH showed $76.4 \mathrm{~nm}$ or $51.6 \mathrm{~nm}$ in particle size, and $48.4 \mathrm{mV}$ or $39.9 \mathrm{mV}$ in $\zeta$-potential (Table 1). The ternary complexes of pDNA with PLL and PLH showed similar particle size and $\zeta$-potential. The binary and ternary complexes can include pDNA stably (Fig. 1). Addition of PLH to pDNA and PLL did not greatly affect the manufacturing characteristics of the particles.

As projected, the gene expressions of ternary complexes which replaced PLL with PLH at rates of 25\% and 50\% (PLL6-PLH2 and PLL4-PLH4 complexes) were 42.0-fold and 35.7-fold higher than that of binary complexes of pDNA and PLL (PLL8-PLL0 complexes), respectively (Fig. 2A). On the other hand, binary complexes with PLH (PLL0-PLH8 complexes) and ternary complexes which replaced PLL with PLH at a rate of 75\% (PLL2-PLH6 complexes) showed lower luciferase activities. PLL0-PLH8 complexes showed very low uptake by cells (Fig. 2B, iii). Therefore, the low gene expression of PLL0-PLH8 complexes was mainly due to the low uptake of PLL0-PLH8. Zhao et al. reported that synthetic histidinylated PLL based gene vector showed unstable and low gene expression by introducing many histidine group. ${ }^{30}$ Introducing of histidine group to the PLL complexes reduced cationic characteristics of the complexes. The low uptake of PLL0-PLH8 may be caused by slight low cationic surface and peculiar structure of PLH. 
The mixed solution of PLL and PLH showed higher buffering capacity between pH 5 and 7 than PLL solution (Fig. 2B). PEI and histidylated polymers showed similar titration profiles through neutral to weakly acidic conditions. ${ }^{31-33}$ The endosomal escape mechanism of pDNA medicated by PEI was supposed to occur via the pH-buffering effect. The imidazole group of histidine had $\mathrm{pKa} 6.0^{34}$ and thus became cationic in slightly acidic conditions.

Furthermore, the transfection efficiency of PLL6-PLH2 complexes was markedly reduced when cells were incubated in the presence of bafilomycin $A_{1}$, a specific inhibitor of the ATPase responsible for the accumulation of protons inside the endosome lumen. ${ }^{35,36}$ On the other hand, strong cellular uptake of both the PLL8-PLH0 and PLL6-PLH2 complexes was observed during experiments involving complexes containing pDNA labelled with YOYO-1. These results indicate that the addition of PLH to PLL complexes induced endosomal escape by enhancing the pH-buffering effect to increase the gene expression.

The strong interaction of cationic complexes to anionic cell membrane often cause to strong cytotoxicity and blood agglutination. In fact, cationic complexes such as binary complexes with PLL and ternary complexes of PLL6-PLH2 complexes showed strong cytotoxicity and blood agglutination (Fig. 5). One promising approach to overcoming 
such cytotoxicity is the addition of anionic polymers to cationic complexes. For example, we previously found that $\gamma$-PGA decreased the toxicity of cationic compounds. $^{23}$ Therefore, we developed quaternary complexes of pDNA by the addition of $\gamma$-PGA to PLL6-PLH2 complexes.

The addition of anionic polymers often caused the release of pDNA containing the complexes. However, the addition of PGA did not release pDNA from quaternary complexes (Fig. 3). Complexes with charge ratios of 8 or 10 for $\gamma$-PGA to pDNA (PLL6-PLH2-PGA8 and PLL6-PLH2-PGA10 complexes) showed a particle size of around $100 \mathrm{~nm}$ with anionic surface charge, suggesting stable anionic particles. The anionic surface charge of complexes largely reduced cell surface interaction and blood agglutination to decrease cytotoxicity and blood toxicity. In fact, the change in the surface charge from positive to negative markedly decreased cytotoxicity and blood agglutination (Fig. 5). The addition of alginic acid and anionic PEG derivatives to cationic complexes was also demonstrated to reduce cytotoxicity and blood agglutination by changing the surface charge..$^{37,38}$

On the other hand, the anionic surface charge of particles also reduced gene expression by decreasing cell surface interactions. We prepared various anionic coating complexes and most could not be taken up well by cells and showed low gene 
expression. $^{23}$ Among these anionic polymers, however, we discovered that $\gamma$-PGA was a rare constituent which was taken up by cells and showed high gene expression. ${ }^{23}$ In fact, all quaternary complexes coated with $\gamma$-PGA showed high gene expression comparable to ternary complexes (PLL6-PLH2 complexes) (Fig. 4A). These transfection efficiencies were as great as the commercially available transfection reagent, lipofectin $\left(1.5 \times 10^{8} \mathrm{RLU} / \mathrm{mg} \text { protein }\right)^{39}$, although their transgene efficacies were lower than those of the binary complexes of pDNA with PAMAM $\left(1.4 \times 10^{9} \mathrm{RLU} / \mathrm{mg}\right.$ protein) or PEI (2.2 × $10^{10} \mathrm{RLU} / \mathrm{mg}$ protein). However, PAMAM and PEI are not biodegradable and exhibit strong toxicity ${ }^{40,41}$, therefore they are not available yet for clinical use. The present quaternary complexes are biodegradable and are constructed by self-assembly. They showed no cytotoxicity and blood toxicity. The complexes may be easily manufactured with sterilization and expected for clinical use.

In the previous report, we confirmed that $\gamma$-PGA-coated complex was taken up by an energy-dependent process and $\gamma$-PGA-specific receptor-mediated pathway using B16F10 cells. ${ }^{23}$ Then, to clarify the mechanisms responsible for the uptake of the complexes, we performed an inhibition study with various endocytotic inhibitors, such as chlorpromazine (CPZ) for clathrin-mediated endocytosis, genistein for caveolae-mediated endocytosis, and amiloride for macropinocytosis. ${ }^{4}$ The transgene 
efficiency of PLL6-PLH2-PGA8 complexes was markedly decreased in the presence of amiloride, suggesting that macropinocytosis must be the primary route of transfection for PLL-PLH-PGA complexes (Fig. 4B). Furthermore, the transfection efficiency of PLL6-PLH2-PGA8 complexes was markedly inhibited by bafiromycin A1. This result indicated that quaternary complexes have the pH-buffering efficiency of PLH.

For the clinical gene therapy, not only high transfection efficiency but also biocompatibility for frequent dosing is indispensable. PLL6-PLH2-PGA8 complexes showed high gene transfection efficiency without cytotoxicity and agglutination, indicating their utility as a clinical gene delivery system. We therefore determined the in vivo transgene efficiencies of PLL6-PLH2-PGA8 complexes. PLL6-PLH2-PGA8 complexes showed high gene expression in the spleen $\left(3.4 \times 10^{5} \mathrm{RLU} / \mathrm{mg}\right.$ protein) after intravenous injection (Fig. 6). Sutherland et al. reported that poly- $\gamma$-D-glutamic acid $(\gamma \mathrm{DPGA})$, which is a capsular component of Bacillus anthracis, was mainly accumulated in the spleen and liver after intravenous injection into mice. ${ }^{42,43}$ In particular, $\gamma$ DPGA was located in red pulp in the spleen and Kupffer cells and sinusoidal endothelial cells in the liver. PLL6-PLH2-PGA8 complexes may be taken up by splenic macrophage through a similar mechanism of $\gamma$ DPGA. Various $\gamma$-PGA-coated complexes were reported to be accumulated in the limbic system of the spleen and 
showed gene expression after their intravenous injection into mice. ${ }^{44}$ It was reported that the gene vector which showed high gene expression in the spleen was an effective DNA vaccine for malaria. ${ }^{45}$

Thus, we successfully modified binary complexes of pDNA with PLL by the addition of PLH and $\gamma$-PGA to develop quaternary complexes with high gene expression and no toxicity. 


\section{ACKNOWLEDGEMENT}

This study was supported in part by a Grant-in-Aid for Scientific Research from the Ministry of Education, Culture, Sports, Science, and Technology, Japan. 


\section{REFERENCES}

1. Canine BF, Hatefi A 2010. Development of recombinant cationic polymers for gene therapy research, Adv Drug Deliv Rev 62: 1524-1529.

2. Tros de Ilarduya C, Sun Y, Duzgunes N. 2010. Gene delivery by lipoplexes and Polyplexes. Eur J Pharm Sci 40: 159-170.

3. Boussif O, Lezoualc’h F, Zanta MA, Mergny MD, Scherman D, Demeneix B, Behr JP 1995. A versatile vector for gene and oligonucleotide transfer into cells in culture and in vivo: polyethylenimine. Proc Natl Acad Sci U.S.A. 92: 7297-7301.

4. Khalil IA, Kogure K, Akita H, Harashima H 2006. Uptake pathways and subsequent intracellular trafficking in nonviral gene delivery. Pharmacol Rev 58: 32-45.

5. Wagner E, Cotton M, Foisner R, Birnstiel ML 1991. Transferrin-polycation-DNA complexes: the effect of polycations on the structure of the complex and DNA delivery to cells. Proc Natl Acad Sci U.S.A. 88: 4255-4259.

6. Farrell LL, Pepin J, Kucharski C, Lin X, Xu Z, Uludag H 2007. A comparison of the effectiveness of cationic polymers poly-l-lysine (PLL) and polyethylenimine (PEI) for non-viral delivery of plasmid DNA to bone marrow stromal cells (BMSC). Eur J Pharm Biopharm 65: 388-397.

7. Yamagata M, Kawano T, Shiba K, Mori T, Katayama Y, Niidome T 2007. Structural advantage of dendritic poly(L-lysine) for gene delivery into cells. Bioorg Med Chem 
15: 526-532.

8. Sonawane ND, Szoka FC, Verkman A 2003. Chloride accumulation and swelling in endosomes enhances DNA transfer by polyamine-DNA polyplexes. J Biol Chem 278: 44826-44831.

9. Tseng SJ, Tang SC, Zeng YF, Cherng JY, Shih MF 2005. Structural characterization and buffering capacity in relation to the transfection efficiency of biodegradable polyurethane. Bioconjug Chem 16: 1375-1381.

10. Shigeta K, Kawakami S, Higuchi Y, Okuda T, Yagi H, Yamashita F, Hashida M 2007. Novel histidine-conjugated galactosylated cationic liposomes for efficient hepatocyte-selective gene transfer in human hepatoma HepG2 cells. J Controll Release 118: $262-270$

11. Abe H 2000. Role of histidine-related compounds as intracellular proton buffering constituents in vertebrate muscle. Biochemistry (Mosc.) 65: 757-765.

12. Kichler A, Leborgne C, März J, Danos O, Bechinger B 2003. Histidine-rich amphipathic peptide antibiotics promote efficient delivery of DNA into mammalian cells. Proc Natl Acad Sci U.S.A. 100: 1564-1568.

13. Pichon C, Gonçalves C, Midoux P 2001. Histidine-rich peptides and polymers for nucleic acids delivery. Adv Drug Delivery Rev 53: 75-94. 
14. Midoux P, Monsigny M 1999. Efficient gene transfer by histidylated

polylysine/pDNA complexes. Bioconjug Chem 10: 406-411.

15. Swami A, Aggarwal A, Pathak A, Patnaik S, Kumar P, Singh Y, Gupta KC 2007. Imidazolyl-PEI modified nanoparticles for enhanced gene delivery. Int J Pharm 335: 180-192.

16. AokiY, Hosaka S, Kawa S, Kiyosawa K 2001. Potential tumor-targeting peptide vector of histidylated oligolysine conjugated to a tumor-homing RGD motif. Cancer Gene Ther 8: 783-787.

17. Dekie L, Toncheva V, Dubruel P, Schacht EH, Barrett L, Seymour LW 2000. Poly-L-glutamic acid derivatives as vectors for gene therapy. J Control Release 65: 187-202.

18. D. Fischer, T. Bieber, Y. Li, H.P. Elsasser, T. Kissel 1999. A novel non-viral vector for DNA delivery based on low molecular weight, branched polyethylenimine: effect of molecular weight on transfection efficiency and cytotoxicity. Pharm Res 16: 1273-1279.

19. Ogris M, Brunner S, Schuller S, Kircheis R, Wagner E 1999. PEGylated DNA/transferrin-PEI complexes: reduced interaction with blood components, extended circulation in blood and potential for systemic gene delivery. Gene Ther 6: 595-605. 
20. Ogris M, Wagner E 2002. Targeting tumors with non-viral gene delivery systems.

Drug Discov Today 7: 479-485.

21 Trubetskoy VS, Wong SC, Subbotin V, Budker VG, Loomis A, Hagstrom JE, Wolff JA 2003.

Recharging cationic DNA complexes with highly charged polyanions for in vitro and in vivo gene delivery. Gene Ther 10: 261-271.

22. Ito T, Iida-Tanaka N, Koyama Y 2008. Efficient in vivo gene transfection by stable DNA/PEI complexes coated by hyaluronic acid. J Drug Target 16: 276-281.

23. Kurosaki T, Kitahara T, Fumoto S, Nishida K, Nakamura J, Niidome T, Kodama Y, Nakagawa H, To H, Sasaki H 2009. Ternary complexes of pDNA, polyethylenimine, and gamma-polyglutamic acid for gene delivery systems. Biomaterials 30: 2846-2853.

24. Kurosaki T, Kitahara T, Kawakami S, Nishida K, Nakamura J, Teshima M, Nakagawa H, Kodama Y, To H, Sasaki H 2009. The development of a gene vector electrostatically assembled with a polysaccharide capsule. Biomaterials 30: 4427-4434. 25. Kabanov AV 1999. Taking polycation gene delivery systems from in vitro to in vivo. Pharm Sci Technol Today 2: 365-372.

26. Godbey WT, Wu KK, Mikos AG 1999. Poly(ethylenimine) and its role in gene delivery. J Control Release 60: 149-160.

27. M.X. Tang, F.C. Szoka 1997. The influence of polymer structure on the 
interactions of cationic polymers with DNA and morphology of the resulting complexes.

Gene Ther 4: 823-832 .

28. Cherng JY 2009. Investigation of DNA spectral conformational changes and polymer buffering capacity in relation to transfection efficiency of DNA/polymer complexes. J Pharm Pharm Sci 12: 346-356.

29. Abe H, Dobson GP, Hoeger U, Parkhouse WS 1985. Role of histidine-related compounds to intracellular buffering in fish skeletal muscle. Am J Physiol 249:

R449-454.

30. Zhao GX, Tanaka H, Kim CW, Li K, Funamoto D, Nobori T, Nakamura Y, Niidome T, Kishimura A, Mori T, Katayama Y 2014. Histidinylated poly-L-lysine-based vectors for cancer-specific gene expression via enhancing the endosomal escape. $\mathrm{J}$ Biomater Sci Polym Ed. 25: 519-534.

31. Thomas M, Lu JJ, Ge Q, Zhang C, Chen J, Klibanov AM 2005. Full deacylation of polyethylenimine dramatically boosts its gene delivery efficiency and specificity to mouse lung. Proc Natl Acad Sci U. S. A. 102: 5679-5684.

32. Benns JM, Choi JS, Mahato RI, Park JS, Kim SW 2000. pH-sensitive cationic polymer gene delivery vehicle: N-Ac-poly(l-histidine)-graft-poly(l-lysine) comb shaped polymer. Bioconjug Chem 11: 637-645. 
33. Leng Q, Mixson AJ 2005. Modified branched peptides with a histidine-rich tail enhance in vitro gene transfection. Nucleic Acids Res 33 e40.

34. Patchornic A, Berger A, Katchalski E 1957. Poly-l-histidine. J Am Chem Soc 79: $5227-5236$.

35. Furuchi T, Aikawa K, Arai H, Inoue K 1993. Bafilomycin A1, a specific inhibitor of vacuolar-type $\mathrm{H}(+)$-ATPase, blocks lysosomal cholesterol trafficking in macrophages.

J Biol Chem 268: 27345-27348.

36. Singh RS, Gonçalves C, Sandrin P, Pichon C, Midoux P, Chaudhuri A 2004. On the gene delivery efficacies of $\mathrm{pH}$-sensitive cationic lipids via endosomal protonation: a chemical biology investigation. Chem Biol 11: 713-723.

37. Jiang G, Min SH, Kim MN, Lee DC, Lim MJ, Yeom YI 2006. Alginate/PEI/DNA polyplexes: a new gene delivery system. Yao Xue Xue Bao 41: 439-445.

38. Maruyama K, Iwasaki F, Takizawa T, Yanagie H, Niidome T, Yamada E, Ito T, Koyama Y 2004. Novel receptor-mediated gene delivery system comprising plasmid/protamine/sugar-containing polyanion ternary complex. Biomaterials 25: 3267-3273.

39. Kurosaki T, Morishita T, Kodama Y, Sato K, Nakagawa H, Higuchi N, Nakamura T, Hamamoto T, Sasaki H, Kitahara T. 2010. Nanoparticles electrostatically coated with 
folic acid for effective gene therapy. Mol Pharm. 8: 913-919.

40. Moghimi SM, Symonds P, Murray JC, Hunter AC, Debska G, Szewczyk AA 2005.

Two-stage poly(ethylenimine)-mediated cytotoxicity: implications for gene

transfer/therapy. Mol Ther 11: 990-995.

41. Mukherjee SP, Lyng FM, Garcia A, Davoren M, Byrne HJ. 2010. Mechanistic studies of in vitro cytotoxicity of poly(amidoamine) dendrimers in mammalian cells.

Toxicol Appl Pharmacol 248: 259-268.

42. Sutherland MD, Thorkildson P, Parks SD, Kozel TR. 2008. In vivo fate and distribution of poly-gamma-D-glutamic acid, the capsular antigen from Bacillus anthracis. Infect Immun 76: 899-906.

43. Sutherland MD, Kozel TR. 2009. Macrophage uptake, intracellular localization, and degradation of poly-gamma-D-glutamic acid, the capsular antigen of Bacillus anthracis. Infect Immun 77: 532-538.

44. Kurosaki T, Kodama Y, Muro T, Higuchi N, Nakamura T, Kitahara T, Miyakoda M, Yui K, Sasaki H 2013. Secure splenic delivery of plasmid DNA and its application to DNA vaccine. Biol Pharm Bull 36: 1800-1806.

45. Cherif MS, Shuaibu MN, Kurosaki T, Helegbe GK, Kikuchi M, Yanagi T, Tsuboi T, Sasaki H, Hirayama K 2011. Immunogenicity of novel nanoparticle-coated MSP-1 
C-terminus malaria DNA vaccine using different routes of administration. Vaccine 29:

9038-9050. 


\section{FIGURE LEGENDS}

Figure 1.

Electrophoretic migration of pDNA in binary and ternary complexes through agarose gel. PLL-PLH complexes were loaded onto an agarose gel, and electrophoresis was carried out. The retardation of pDNA was visualized using ethidium bromide.

Figure 2.

(A) In vitro transfection efficiencies of PLL-PLH complexes. B16-F10 cells were transfected with various complexes containing pCMV-Luc. Twenty-two hours after transfection, cells were lysed and luciferase activities were determined. Each value is the mean \pm S.E. $(n=3) . \quad *$ P $<0.05$ vs. PLL8-PLH0 complex.

(B) Fluorescence microscopy images of transfected B16-F10 cells. B16-F10 cells were transfected with YOYO-1 labeled pCMV-Luc-containing complexes. Twenty-two hours after the cells had been transfected, the uptake of PLL8-PLH0 (i), PLL6-PLH2 (ii), and PLL0-PLH8 complexes (iii) was assessed (200 × magnification).

(C) Buffering capacity of PLL and PLL-PLH solution. $\mathrm{NaOH} \mathrm{0.01} \mathrm{M}$ was used to titrate the PLL solution or mixed solution of PLL and PLH. Molar ratio of PLL to PLH in mixed solution was the same as for PLL6-PLL2 complexes. 0 : PLL, $\bigcirc$ : 


\section{PLL-PLH}

(D) PLL6-PLL2 complexes were transfected in medium with bafiromycin $\mathrm{A}_{1}$.

Twenty-two hours after transfection, cells were lysed and luciferase activities were determined. Each value is the mean \pm S.E. $(n=3) . \quad * \mathrm{P}<0.05$ vs. control.

Figure 3.

Electrophoretic migration of pDNA in quaternary complexes through agarose gel.

PLL-PLH-PGA complexes were loaded onto an agarose gel, and electrophoresis was carried out. The retardation of pDNA was visualized using ethidium bromide.

Figure 4.

(A) In vitro transfection efficiencies of PLL-PLH-PGA complexes. B16-F10 cells were transfected with various complexes containing pCMV-Luc. Twenty-two hours after transfection, cells were lysed and luciferase activities were determined. Each value is the mean \pm S.E. $(n=3) . \quad * \mathrm{P}<0.05$ vs. PLL6-PLH2 complex. (B) Influence of endocytotic inhibitors on the transfection efficiency of PLL6-PLH2-PGA8 complexes. PLL6-PLL2-PGA8 complexes were transfected in medium with various endocytotic inhibitors. Twenty-two hours after transfection, cells 
were lysed and luciferase activities were determined. Each value is the mean \pm S.E. ( $n$ $=3)$.

(C) Influence of endocytotic inhibitors bafiromycin $A_{1}$ on the transfection efficiency of PLL6-PLH2-PGA8 complexes. PLL6-PLL2-PGA8 complexes were transfected in medium with bafiromycin $A_{1}$. Twenty-two hours after transfection, cells were lysed and luciferase activities were determined. Each value is the mean \pm S.E. $(n=3) . \quad *$ P $<0.05$ vs. control.

Figure 5.

(A) Cytotoxicity of the complexes on B16-F10 cells. The viability of cells treated with each complex was measured by the WST-1 assay. Cell viability was measured $22 \mathrm{~h}$ after cells had been incubated with the complexes for $2 \mathrm{~h}$. Data are the percentage to untreated cells. $\quad$ Each value is the mean \pm S.E. $(n=8) . \quad * \mathrm{P}<0.05$ vs. control. (B) Agglutination of complexes with erythrocytes. Each complex was added to erythrocytes, and agglutination was observed by microscopy (400 × magnification). (i) PBS, (ii) PLL8-PLL0 complexes, (iii) PLL6-PLH2 complexes, (IV) PLL6-PLH2-PGA8 complexes. 
Figure 6.

In vivo transgene efficiencies of the complexes in mice. The complexes were injected intravenously into mice (40 $\mu$ g DNA per mouse). Six hours after the injection, mice were sacrificed and each organ was dissected to quantify luciferase activity.

PLL8-PLL0 complex ( $\square$ ), PLL6-PLH2 complex ( $\square$ ) and PLL6-PLH2-PGA8 complex

( $\square)$. Each value is the mean \pm S.E. $(n=3) . \quad * P<0.05$, vs PLL complex, $\uparrow P<0.05$, vs PLL-PLH complex. 
Fig. 1

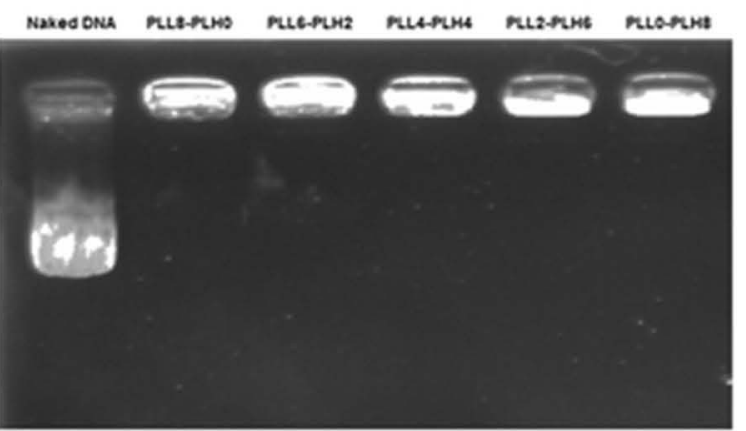

$36 \times 27 \mathrm{~mm}(300 \times 300 \mathrm{DPI})$ 
Fig. 2
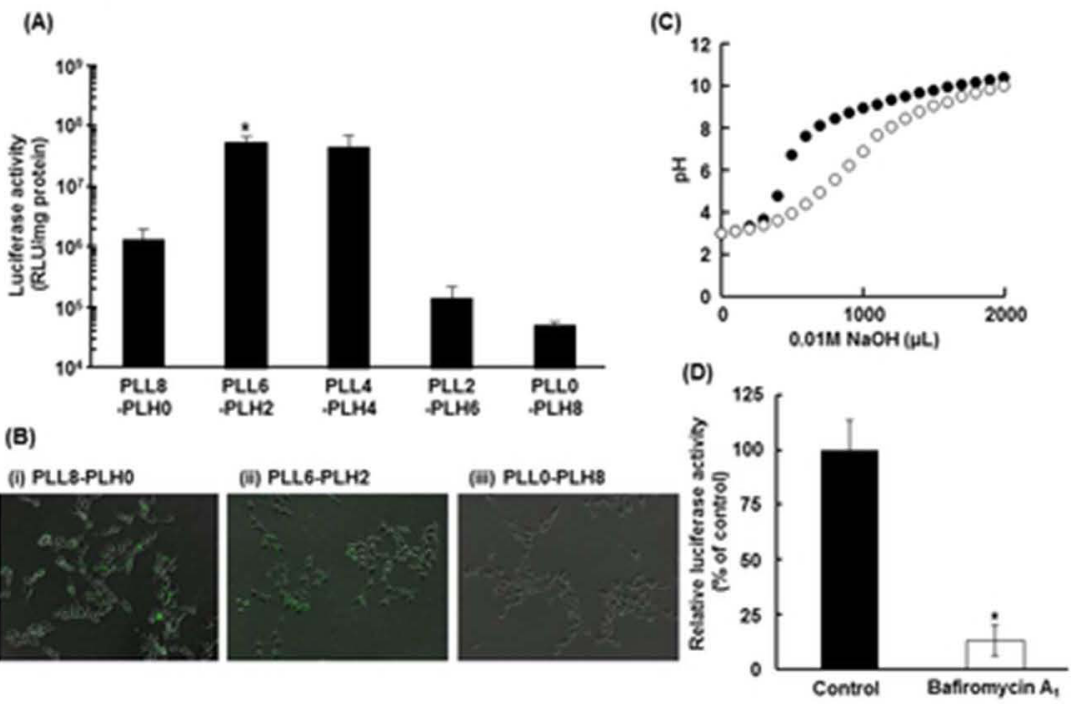

(A) In vitro transfection efficiencies of PLL-PLH complexes. B16-F10 cells were transfected with various complexes containing PCMV-Luc. Twenty-two hours after transfection, cells were lysed and luciferase activities were determined. Each value is the mean \pm S.E. $(n=3)$. ${ }^{*} P<0.05$ vs. PLL8-PLH0 complex. (B) Fluorescence microscopy images of transfected B16-F10 cells. B16-F10 cells were transfected with YOYO-1 labeled pCMV-Luc-containing complexes. Twenty-two hours after the cells had been transfected, the uptake of PLL8-PLHO (i), PLL6-PLH2 (ii), and PLLO-PLH8 complexes (iii) was assessed (200 $\times$ magnification).

(C) Buffering capacity of PLL and PLL-PLH solution. $\mathrm{NaOH} 0.01 \mathrm{M}$ was used to titrate the PLL solution or mixed solution of PLL and PLH. Molar ratio of PLL to PLH in mixed solution was the same as for PLL6-PLL2 complexes. •: PLL, $\square 0:$ PLL-PLH

(D) PLL6-PLL2 complexes were transfected in medium with bafiromycin A1. Twenty-two hours after transfection, cells were lysed and luciferase activities were determined. Each value is the mean \pm S.E. $(n=$ 3). ${ }^{*} \mathrm{P}<0.05$ vs. control. $36 \times 27 \mathrm{~mm}(300 \times 300 \mathrm{DPI})$ 
Fig. 3

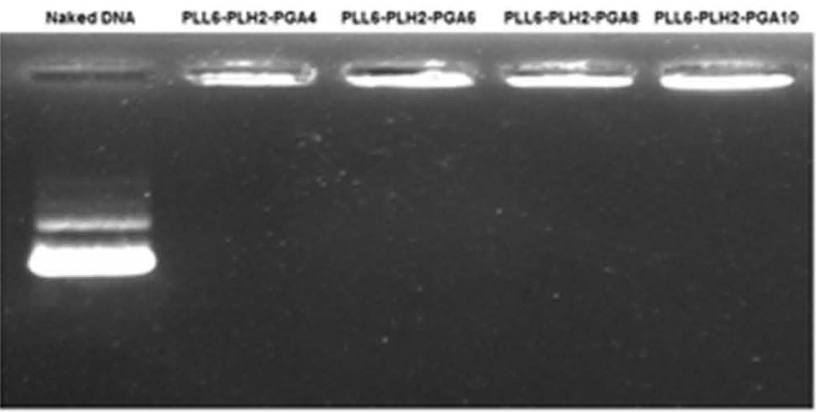

$36 \times 27 \mathrm{~mm}(300 \times 300 \mathrm{DPI})$ 
Fig. 4
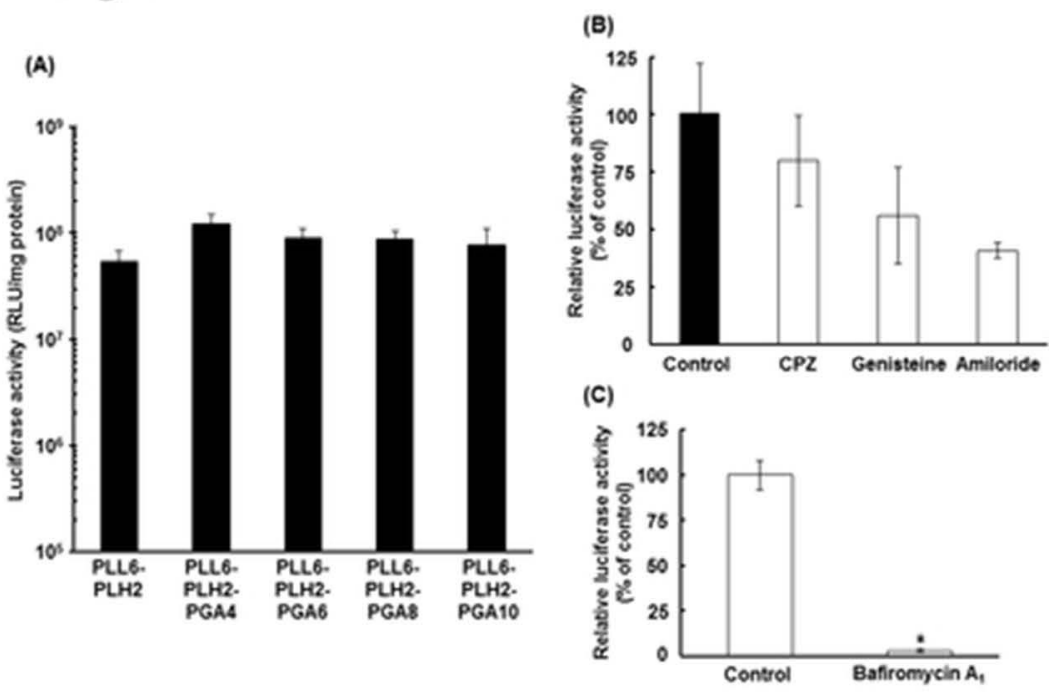

$36 \times 27 \mathrm{~mm}(300 \times 300 \mathrm{DPI})$ 
Fig. 5

(A)

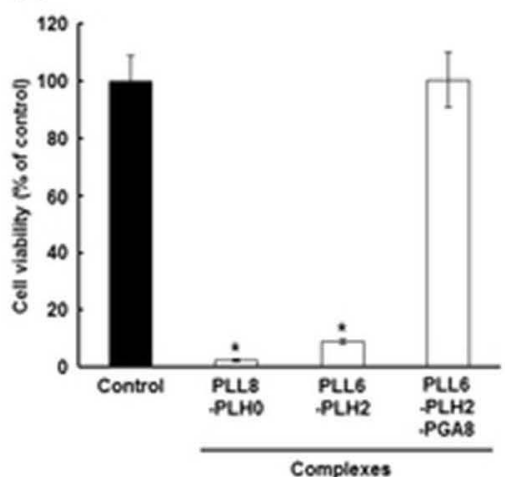

(B)
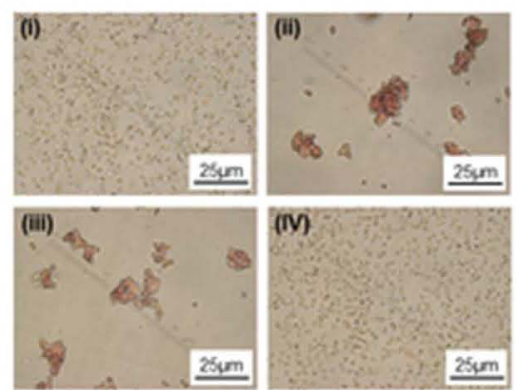

(iv)

$36 \times 27 \mathrm{~mm}(300 \times 300 \mathrm{DPI})$ 
Fig. 6

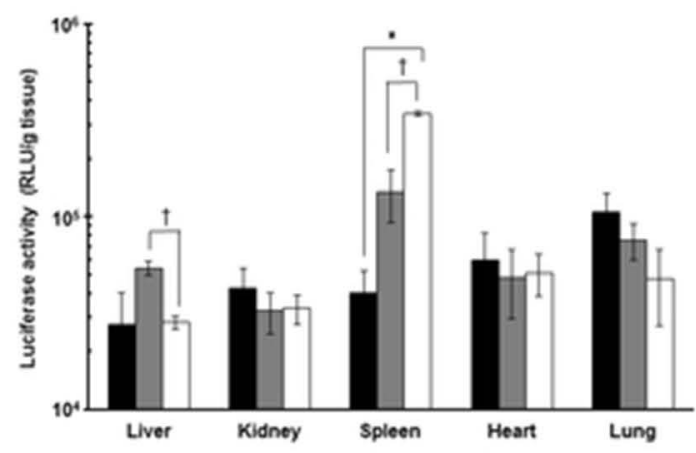

$36 \times 27 \mathrm{~mm}(300 \times 300 \mathrm{DPI})$ 


\section{Tables}

Table 1

Particle size and $\zeta$-potential of binary and ternary complexes.

\begin{tabular}{ccc}
\hline Complexes & 万-potential $(\mathrm{mV})$ & Size $(\mathrm{nm})$ \\
\hline PLL8-PLH0 complex & $48.4 \pm 0.6$ & $76.4 \pm 22.1$ \\
PLL6-PLH2 complex & $42.3 \pm 0.1$ & $64.1 \pm 8.3$ \\
PLL4-PLH4 complex & $41.3 \pm 0.8$ & $70.1 \pm 4.7$ \\
PLL2-PLH6 complex & $40.3 \pm 0.8$ & $62.7 \pm 4.0$ \\
PLL0-PLH8 complex & $39.9 \pm 0.5$ & $51.6 \pm 8.8$ \\
\hline & & Each data is mean \pm S.D. $(\mathrm{n}=3)$
\end{tabular}


Table 2

Particle size and $\zeta$-potential of quaternary complexes.

\begin{tabular}{ccc}
\hline Complexes & $\zeta$-potential $(\mathrm{mV})$ & Size $(\mathrm{nm})$ \\
\hline PLL6-PLH2-PGA4 complex & $0.5 \pm 0.3$ & undetectable \\
PLL6-PLH2-PGA6 complex & $-7.4 \pm 0.1$ & $272.5 \pm 1.6$ \\
PLL6-PLH2-PGA8 complex & $-22.0 \pm 0.5$ & $106.9 \pm 6.5$ \\
PLL6-PLH2-PGA10 complex & $-28.6 \pm 0.6$ & $120.0 \pm 9.0$ \\
\hline
\end{tabular}

